\title{
High miss rate for gastric superficial cancers at endoscopy: what is necessary for gastric cancer screening and surveillance using endoscopy?
}

\section{다(1)우우}

\author{
Authors \\ Masau Sekiguchi ${ }^{1,2,3}$, Ichiro Oda ${ }^{1}$ \\ Institutions \\ 1 Endoscopy Division, National Cancer Center Hospital, \\ Tokyo, Japan \\ 2 Cancer Screening Center, National Cancer Center \\ Hospital, Tokyo, Japan \\ 3 Division of Screening Technology, Center for Public \\ Health Sciences, National Cancer Center, Tokyo, Japan
}

\author{
Bibliography \\ DOI https://doi.org/10.1055/s-0043-112245 | \\ Endoscopy International Open 2017; 05: E727-E728 \\ (c) Georg Thieme Verlag KG Stuttgart · New York \\ ISSN 2364-3722
}

Corresponding author

Masau Sekiguchi, MD, PhD, Endoscopy Division, National

Cancer Center Hospital, 5-1-1 Tsukiji, Chuo-ku, Tokyo, 104-

0045, Japan

Fax: $+81-3-35423815$

masekigu@ncc.go.jp
Gastric cancer is an important screening target, particularly in countries with high gastric cancer incidence and mortality, such as East Asian countries. Endoscopy plays an increasingly important role in screening and diagnosis of gastric cancer. For instance, in Japan, endoscopy has been recently accepted as a primary tool in population-based gastric cancer screening [1]. Endoscopy is also a vehicle for modern gastric cancer treatments, such as endoscopic submucosal dissection (ESD) [24]. Although advanced gastric cancer still has a poor prognosis, patients with early-stage disease treated with ESD are likely to have favorable outcomes [3-5]. For that reason, early detection of gastric cancer is important and endoscopy is a critical tool for its early detection, diagnosis, and treatment.

Endoscopy is considered to be the most powerful tool for detecting gastric cancer [6]; however, it does not always find existing cancers, and its miss rate has been examined in several studies. In 2014, a meta-analysis by Menon et al., published in Endoscopy International Open, examined how commonly upper gastrointestinal (GI) cancer is missed at endoscopy [7]. The authors defined missed cancers as cancers that had not been diagnosed by endoscopies performed within 3 years before diagnosis, based on the hypothesis that mucosal cancer has a 2 -3year doubling time [8]. The study concluded that $11.3 \%$ of upper $\mathrm{Gl}$ cancers were missed at endoscopy performed within 3 years before the diagnosis, and showed that $0.25 \%$ of all the endoscopic procedures missed existing upper Gl cancers. Although these data are important and informative, interpreting the results can be difficult because the examined upper GI can- cers included both gastric cancers and esophageal cancers, and also cancers at various stages. Considering the heterogeneity of these lesions, the limitations of this study (particularly the definition of missed cancer based on a specific interval between diagnosis and preceding endoscopy) cannot be ignored; further data on missed cancers from studies performed in different study methods are therefore needed.

In this regard, the paper by Shimodate et al. from a Japanese institution published in Endoscopy International Open 2017, provides us with a wider perspective [9]. The authors examined the miss rate of gastric superficial neoplasia (GSN) by reviewing GSN patients' earlier endoscopic records, rather than defining missed cancers by the interval between diagnosis and preceding endoscopy. Although the study has several limitations (e. g. retrospective design, possible selection bias, and lack of full information on preceding endoscopies), five endoscopists in this study actually reviewed the images of the preceding endoscopies and judged the existence of the missed lesions. The study concluded that the miss rate for GSN was $75.2 \%$. The rate seems surprisingly high, but it is necessary to understand the meaning of the value appropriately. This value means not the miss rate of GSN among all endoscopic procedures but the proportion of GSNs overlooked at the preceding endoscopies among all GSNs. In addition, because missed cancers were defined quite differently in this study than in the Menon meta-analysis, their miss rates are difficult to compare [7]. However, even after considering the new definition for miss rate in the Shimodate study and the study's possible selection bias, the 
rate still seems high, probably because the target of the study, GSN, is more difficult to detect than most other upper GI cancers (as the authors mention).

This high rate indicates room for improvement in early detection of gastric cancer. The key factors for such improvement include better training of endoscopists and further advancement of endoscopy. Establishment of an improved training system for endoscopists and quality assurance for endoscopy are essential for more successful gastric cancer screening using endoscopy $[1,10,11]$. With regard to the advancement of endoscopy in detection and diagnosis of upper Gl cancers, image-enhanced endoscopy with/without magnification has been expected to contribute to the improvement $[12,13]$, although further investigation is necessary, particularly for its usefulness in detecting GSN.

The finding of the study that GSN is easily overlooked, even by endoscopists certified by the Japanese Gastrointestinal Endoscopy Society, also makes us realize the necessity of establishing the appropriate surveillance program after endoscopy. In considering the surveillance program, the data on the natural course of missed GSN examined in the study also provide us with important information. The authors examined the clinical course of missed GSNs and showed that only $8.5 \%$ of them progressed to advanced cancer during the mean interval of 39.3 months. Based on the low cumulative incidence rate of progression to advanced cancer $(0.8 \%)$ of missed GSNs at 3 years after endoscopy and increased incidence after 3 years, the authors commented that surveillance endoscopy should be performed at least every 3 years after endoscopy to detect earlystage gastric cancers. The obtained data are important and the comment on surveillance seems logical, but more detailed consideration is necessary regarding the issue of surveillance. From the perspective of limited endoscopy resources, risk stratification of individuals is required, instead of performing surveillance endoscopy within 3 years after the preceding endoscopy for all individuals. Therefore, how to incorporate risk factors for gastric cancer, such as the status of Helicobacter pylori infection [14] and atrophic gastritis [15], into both screening and surveillance programs should be investigated. A large prospective study in consideration of these factors is warranted.

Another aspect of the miss rate of upper GI cancer is that the rate might be used as a quality indicator of upper GI endoscopy $[1,10,11]$. Quality indicators have been well studied in colonoscopy, whereas such indicators for upper GI endoscopy have room for further investigation. In the future investigation of quality indicators for upper GI endoscopy, it will be possible that the miss rates of upper Gl cancers and GSN are examined as candidates for the indicators after discussion on the definition of the miss rates.

In this editorial, we discussed the miss rate for GSNs. Based on the reported high miss rate, improved training for endoscopists, further research and development of endoscopy in detection and diagnosis, and establishment of appropriate surveillance programs are essential. It is also critical that each endoscopist should always consider the risk of overlooking important lesions, such as GSNs, and try to achieve best performance in every upper $\mathrm{GI}$ endoscopic procedure.
Competing interests

\section{None}

References

[1] Hamashima C, Fukao A. Working Group for the quality assurance of endoscopic screening for gastric cancer. Quality assurance manual of endoscopic screening for gastric cancer in Japanese communities. Jpn J Clin Oncol 2016: DOI: 10.1093/jjco/hyw106

[2] Oda I, Gotoda T, Hamanaka H et al. Endoscopic submucosal dissection for early gastric cancer: technical feasibility, operation time and complications from a large consecutive series. Dig Endosc 2005; 17 : $54-58$

[3] Suzuki H, Oda I, Abe S et al. High rate of 5-year survival among patients with early gastric cancer undergoing curative endoscopic submucosal dissection. Gastric Cancer 2016; 19: 198-205

[4] Tanabe S, Ishido K, Matsumoto T et al. Long-term outcomes of endoscopic submucosal dissection for early gastric cancer: a multicenter collaborative study. Gastric Cancer 2017; 20: 45-52

[5] Katai H, Ishikawa T, Akazawa K et al. Registration Committee of the Japanese Gastric Cancer Association. Five-year survival analysis of surgically resected gastric cancer cases in Japan: a retrospective analysis of more than 100,000 patients from the nationwide registry of the Japanese Gastric Cancer Association (2001-2007). Gastric Cancer 2017: DOI: 10.1007/s10120-017-0716-7

[6] Hamashima C, Okamoto M, Shabana M et al. Sensitivity of endoscopic screening for gastric cancer by the incidence method. Int I Cancer 2013; 133: 653-659

[7] Menon S, Trudgill N. How commonly is upper gastrointestinal cancer missed at endoscopy? A meta-analysis Endosc Int Open 2014; 2: E46-50

[8] Fujita S. Biology of early gastric carcinoma. Pathol Res Pract 1978; 163: $297-309$

[9] Shimodate Y, Mizuno M, Doi A et al. Gastric superficial neoplasia: high miss rate but slow progression. Endosc Int Open 2017; 05: $722-$ 726

[10] Park WG, Shaheen NJ, Cohen J et al. Quality indicators for EGD. Gastrointest Endosc 2015; 81: 17-30

[11] Bisschops R, Areia M, Coron E et al. Performance measures for upper gastrointestinal endoscopy: a European Society of Gastrointestinal Endoscopy (ESGE) Quality Improvement Initiative. Endoscopy 2016; 48: $843-864$

[12] Muto M, Minashi K, Yano T et al. Early detection of superficial squamous cell carcinoma in the head and neck region and esophagus by narrow band imaging: a multicenter randomized controlled trial. J Clin Oncol 2010; 28: 1566-1572

[13] Ezoe $\mathrm{Y}$, Muto $\mathrm{M}$, Uedo $\mathrm{N}$ et al. Magnifying narrowband imaging is more accurate than conventional white-light imaging in diagnosis of gastric mucosal cancer. Gastroenterology 2011; 141: 2017-2025

[14] Uemura N, Okamoto S, Yamamoto S et al. Helicobacter pylori infection and the development of gastric cancer. N Engl J Med 2001; 345: 784 789

[15] Masuyama H, Yoshitake N, Sasai T et al. Relationship between the degree of endoscopic atrophy of the gastric mucosa and carcinogenic risk. Digestion 2015; 91: 30 - 36 